\title{
A CASE OF TUBERCULOSIS LYMPHADENITIS MIMICKING GASTRIC TUMOR
}

\author{
Dhafer Hadded $^{1}$, Alia Zouaghi ${ }^{1}$, MERYAM MESBAHI ${ }^{1}$, Yazid Benzarti ${ }^{1}$, Nawel Bellil ${ }^{1}$, \\ Zeineb Benzarti ${ }^{2}$, and Anis Ben Maamer ${ }^{1}$ \\ ${ }^{1}$ Habib Thameur Hospital \\ ${ }^{2}$ University of Tunis El Manar Faculty of Medicine of Tunis
}

June 20, 2021

\begin{abstract}
Tuberculosis of the upper gastrointestinal tract is a rare pathology. Stomach tuberculosis, particularly, can appear as a subepithelial gastric tumor. We describe a case of a Patient with tuberculous intraperitoneal lymphadenitis mimicking the submucosal gastric tumor. An exploratory laparotomy was performed, and the diagnosis was confirmed by frozen section examination.
\end{abstract}

\section{A CASE OF TUBERCULOSIS LYMPHADENITIS MIMICKING GASTRIC TUMOR}

Mesbahi Meryam*(corresponding author):

Resident Surgical Department at Habib Thameur HospitalTunisiaPostal address : 1000Mobile Number : +21629343106Meryam.mesbahi@gmail.com

\begin{abstract}
:
Tuberculosis of the upper gastrointestinal tract is a rare pathology. Stomach tuberculosis, particularly, can appear as a subepithelial gastric tumor. We describe a case of a Patient with tuberculous intraperitoneal lymphadenitis mimicking the submucosal gastric tumor. An exploratory laparotomy was performed, and the diagnosis was confirmed by frozen section examination.
\end{abstract}

Keywords: Gastric tuberculosis, abdominal tuberculosis, Subepithelial tumor, Tuberculous Lymphadenitis

Key clinical message: Our manuscript highlights:

- Gastric tuberculosis is a challenging diagnosis

- Stomach tuberculosis can present as a subepithelial tumor

- It requires histopathological examination to confirm the tuberculous lymphadenitis

Introduction:

Abdominal tuberculosis can affect many organs in the peritoneal cavity such as the gastrointestinal tract, peritoneum, lymph nodes, spleen, and liver ..it can affect one organ or many in combination[1]. Gastrointestinal tuberculosis presentation is varied depending on the site which is involved [2]. Its diagnosis is specifically difficult and this is explained by differential diagnoses that can mimic the various manifestations of gastrointestinal TB, including infectious and noninfectious causes[3]. Tuberculosis of the stomach is the rarest form and it is generally misdiagnosed because it can mimic a gastric tumor[4]. 
We report here a case of mesenteric tuberculous lymphadenitis that had involved the gastric wall and had mimicked a gastric submucosal tumor with no evidence of tuberculosis elsewhere.

\section{Case report:}

A 52-year-old woman, with a history of hypertension, hospitalized in our department of surgery for a 3-month history of epigastric pain and discomfort, and weight loss. She had no fever either respiratory symptoms. Physical examination revealed mild tenderness in the upper of the abdomen, associated with an epigastric palpable and painful mass measuring $4 \mathrm{~cm}$. There is no cervical lymphadenopathy nor hepatosplenomegaly. laboratory data were normal. and there are no abnormalities on a chest Xray

On upper endoscopy showed a submucosal compression or anterior submucosal lesion, erosive anterior gastropathy, and a fistulous orifice located in the bulb. (figure1)

A biopsy of the lesion was performed and concluded to no malignancy and had no evidence of tuberculosis

An abdominal CT scan was performed and showed an exophytic, heterogenous gastric formation with an axial necrotic center measuring $44^{*} 24 \mathrm{~mm}$, associated with multiple tissue nodules of enhanced infra centimeter size after injection of contrast agent evoking peritoneal carcinosis nodules, and coeliomesenteric lymph nodes, one of which has a necrotic center measuring $9 \mathrm{~mm}$ in diameter associated with hepatic hilum lymph nodes. (figure2)

Endoscopic ultrasound demonstrated a rounded lesion of about $30^{*} 26 \mathrm{~mm}$ located in the antrum, hypoechogenic, discretely heterogeneous not vascularized, and it is distant from the gastric wall whose 5 layers appear of normal aspect(figure3). and by positioning the probe next to the bulbar fistulous orifice, we found the lesion with a hypoechogenic center corresponding to the necrosis described at the CT with a hypoechoic fistulous path.

The patient was operated on for exploratory laparotomy due to the prediagnosis of suspected gastric cancer. the surgical findings indicated a bulky mass adjacent to the antrum with posterior development invading the transverse mesocolon, associated with multiple adenopathies of the mesentery, the transverse mesocolon, and the greater omentum, organized inflows. (figure 4)

There is a second mass of $3 \mathrm{~cm}$ located in the small omentum in contact with the left gastric artery, which is probably voluminous adenopathy(figure 4). the biopsy was sent for frozen section examination that concluded to tuberculous intraperitoneal lymphadenitis, so the operation was terminated, gastrotomy was not performed because of the benign nature of the pathology. The patient was administered anti-tuberculosis treatment and was closely monitored.

\section{Discussion :}

the diagnosis of abdominal tuberculosis is always misdiagnosed because of its various clinical manifestations. It is known as a great mimicker especially when it can affect abdominal organs without pulmonary infection, and malignant tumors are the most incriminating as a preoperative diagnosis [2], [5].

Gastric tuberculosis is an extremely rare form whether it was a primary or secondary infection [1]. Debi et al. Explain the reasons for its rarity, such as the bactericidal properties of gastric acid, the scarcity of lymphatic tissue in the gastric wall, and the thick gastric mucosa with an intact stomach [6].

Moreover, mesenteric tuberculose lymphadenitis is extremely rare as a cause of intestinal manifestations involving the gastric wall such in our case[1]. Recording to the literature, A few cases were reported, showing tuberculosis lymphadenopathy mimicking submucosal gastric tumors such as in our case.

Primary gastric tuberculosis (without involving any other organ)is generally located on the antrum or prepyloric region involving the duodenum, and this location is explained by the presence of lymphoid follicles[1], [4]. There are six types of gastric tuberculosis in pathological forms: 1. Tubercular ulcers 2. Miliary tubercles 3. Hypertrophic 4. Tuberculous pyloric stenosis 5. Solitary tuberculoma 6. Tubercular lymphadenitis [2]. 
Clinically, patients generally present nonspecific upper abdominal pain such as epigastric pain, associated with weight loss, anorexia, weakness[4]. So that's why the majority of patients with gastric tuberculosis are often diagnosed after surgery because of the lack of symptoms[1].

Endoscopy is helpful to diagnose this pathology, especially by biopsy results. It can show ulcers, mass, or extrinsic compression[7]. However, in our case, gastric cancer was suspected and the biopsy did not help to confirm our diagnosis. The poor yield of the biopsy is explained by the submucosal lesion that may not reveal granulomas and that is difficult in obtaining tissues[7], [8].

Endoscopic ultrasonography is also very helpful, especially in the case of submucosal lesions or related lymph node enlargement [7]; because it can differentiate between an extrinsic compression and a subepithelial gastric tumor by identifying the relationship between the lesion and the gastric wall[9].

Morphologically, No specific imaging findings can help diagnose tuberculosis rather than malignancy because there are no pathognomonic characteristics showed radiological modalities [5].

Resuming combined radiographic and endoscopic imaging can facilitate an early diagnosis without unnecessary surgical resection, but it is always difficult to have a final diagnosis by endoscopic biopsy so that we can perform a surgical biopsy using frozen section examination[8].

Conclusion:

Abdominal tuberculosis, lymphadenitis tuberculosis present a diagnostic challenge and a dilemma for clinicians, it can mimic a long list of differential diagnoses such as our case of tuberculosis lymphadenitis eroding the gastric wall. In these cases, endoscopy biopsy is the best modality to diagnose it, but it could not be made preoperatively and it requires surgery for diagnosis using intraoperative frozen biopsy.

Conflict of interest for all authors: The authors declare no competing interest.

Funding : None.

Compliance with Ethical Standards: The patient has provided both verbal and written consent for the publication of This article. It was made sure that his identity will be kept a secret at all levels.

Consent: Written informed consent was taken from the patient regarding the publication of this case report. It was made sure that his identity will be kept a secret at all levels. A copy of a written request is available for review if requested.

Acknowledgments: There were no acknowledgments to mention it.

Author contributions : All authors were involved in the researching, writing, and editing of the manuscript.

[1] D. Y. Kim et al. , « Tuberculous mesenteric lymphadenitis involving the gastric wall: case report », Gastrointest. Endosc., vol. 62, no 5, p. 799-802, nov. 2005, doi: 10.1016/j.gie.2005.07.039.

[2] U. Debi, et al. , ii Gastrointestinal Tuberculosis: An overview i¿, Arch. Clin. Med. Case Rep., vol. 04, $\mathrm{n}^{\mathrm{o}}$ 05, 2020, doi: 10.26502 /acmcr.96550269.

[3] C. Lowbridge et al. , ii How can gastro-intestinal tuberculosis diagnosis be improved? A prospective cohort study i¿,BMC Infect. Dis., vol. 20, nº 1, p. 255, dec. 2020, doi: 10.1186/s12879-020-04983-y.

[4] P. Chaudhary, A. Q. Khan, R. Lal, et U. Bhadana, ii Gastric tuberculosis ¿¿, Indian J. Tuberc. , vol. 66, nº3, p. 411-417, juill. 2019, doi: 10.1016/j.ijtb.2018.10.004.

[5] F. M. Abu-Zidan et M. Sheek-Hussein, ii Diagnosis of abdominal tuberculosis: lessons learned over 30 years: pectoral assay ¿¿, World J. Emerg. Surg. , vol. 14, nº 1, p. 33, dec. 2019, doi: 10.1186/s13017-019$0252-3$. 
[6] U. Debi, ii Abdominal tuberculosis of the gastrointestinal tract: Revisited i¿, World J. Gastroenterol. , vol. $20, \mathrm{n}^{\circ}$ 40, p. 14831, 2014, doi: 10.3748/wjg.v20.i40.14831.

[7] J. Shah, P. Maity, P. Kumar-M, A. Jena, P. Gupta, et V. Sharma, ii Gastroduodenal tuberculosis: a case series and a management focused systematic review i. Expert Rev. Gastroenterol. Hepatol. , p. 1-10, sept. 2020, doi: $10.1080 / 17474124.2020 .1816823$.

[8] I. C. Eray et al. , ii Primary gastric tuberculosis mimicking gastric cancer i¿, Turk. J. Surg. , sept. 2014, doi: $10.5152 / \mathrm{UCD} .2014 .2667$.

[9] S. B. Kim, T. N. Kim, et K. H. Kim, ii Tuberculous Lymphadenitis Mimicking Gastric Subepithelial Tumor Diagnosed Using Endoscopic Ultrasound-guided Fine-needle Aspiration ¿¿, Korean J. Helicobacter Up. Gastrointest. Res., vol. 18, $\mathrm{n}^{\circ}$ 1, p. 65, 2018, doi: 10.7704/kjhugr.2018.18.1.65.

Figure 1: Esophagogastroduodenoscopy shows a submucosal compression or anterior submucosal lesion, and a fistulous orifice located in the bulb.

Figure2: Abdominal computed tomography demonstrates an exophytic, heterogenous gastric formation with a necrotic center.

Figure3: Radial endoscopic ultrasound shows round mass Located in the gastric antrum, hypoechoic, discrete heterogeneous, without blood vessels, far from the stomach wall.

Figure 4: the surgical findings indicated: (Figure4A) a bulky mass adjacent to the antrum with posterior development invading the transverse mesocolon / (figure4B) A second mass of $3 \mathrm{~cm}$ in the greater omentum in contact with the left gastric artery, which may be a huge adenopathy

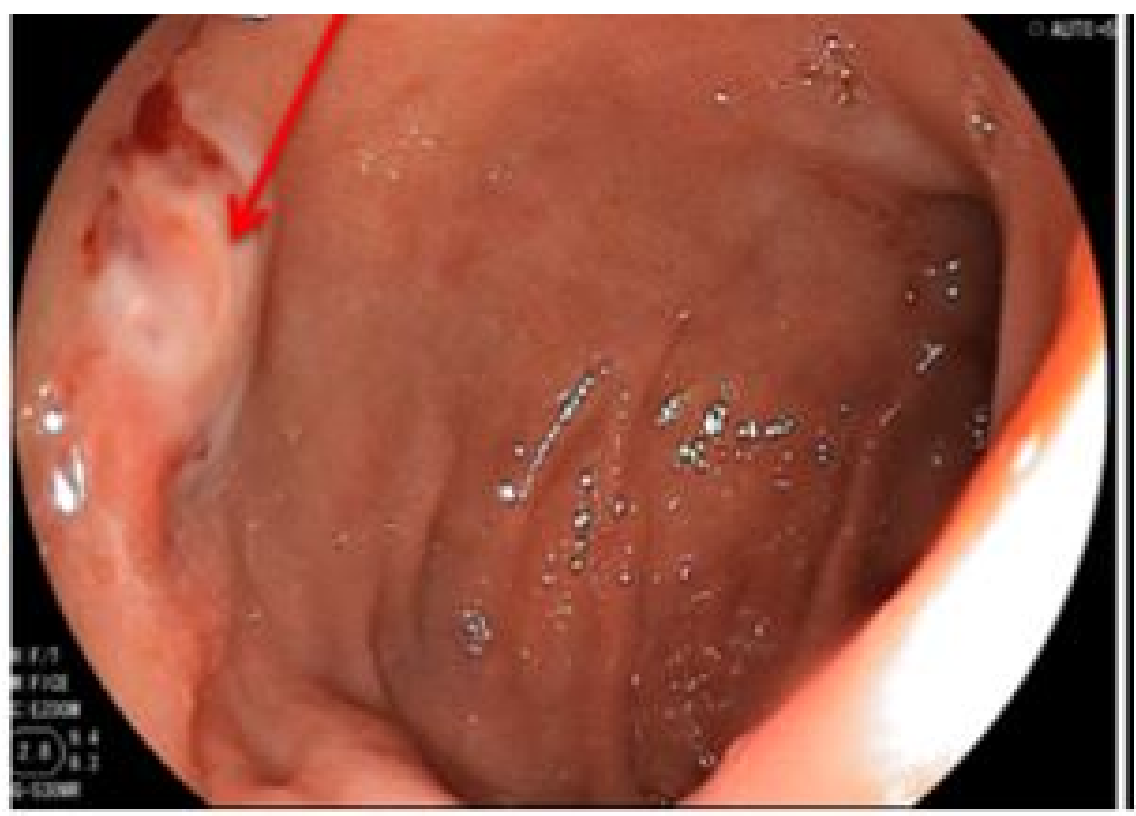



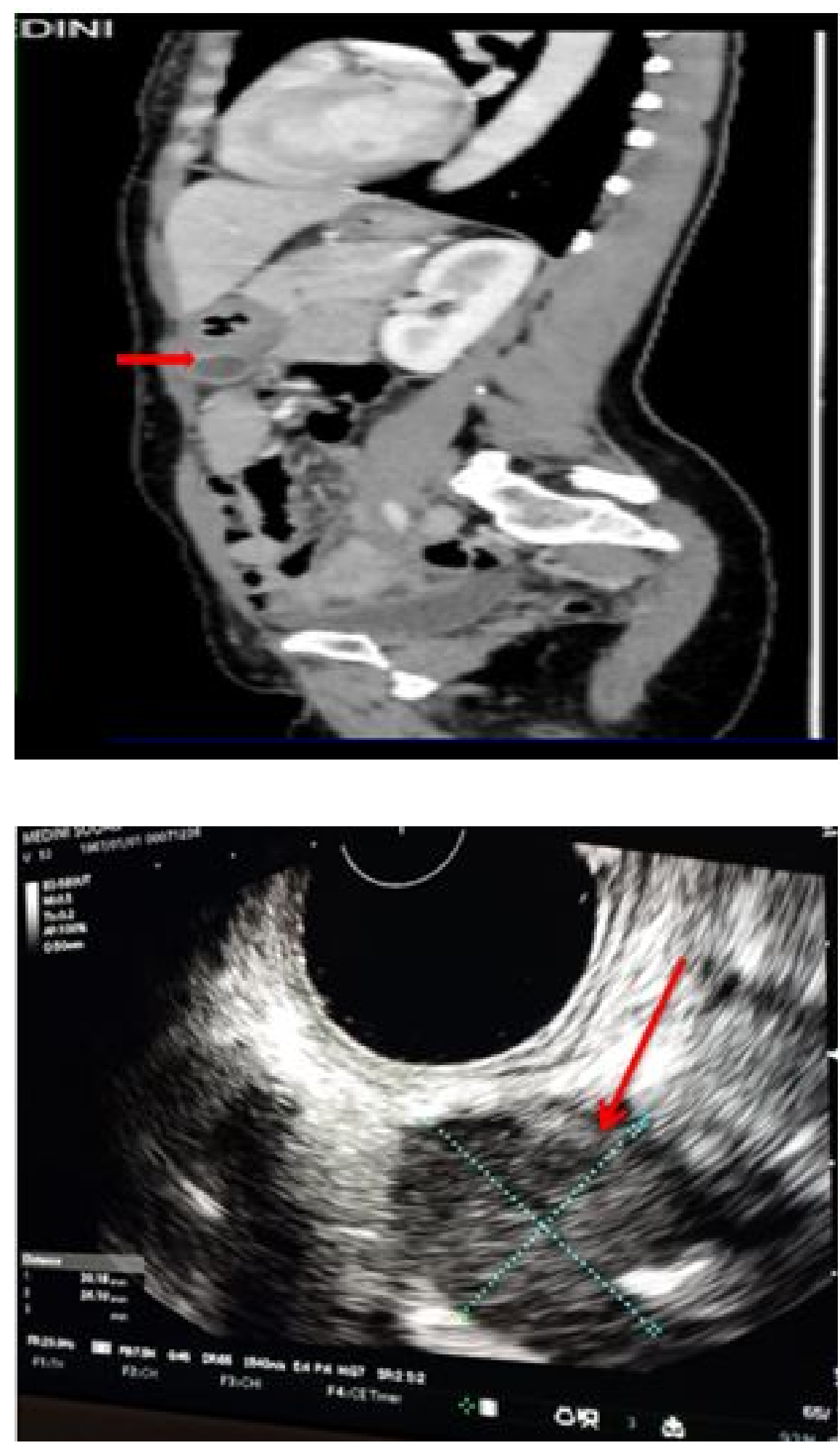

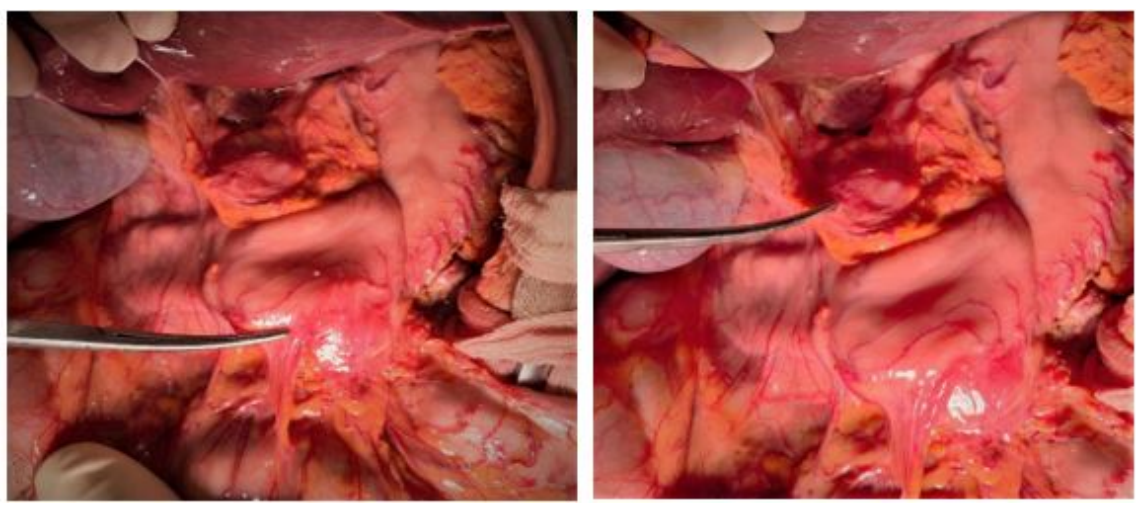